\title{
IUCrJ
}

Volume 2 (2015)

Supporting information for article:

\section{A novel inert crystal delivery medium for serial femtosecond crystallography}

Chelsie E. Conrad, Shibom Basu, Daniel James, Dingjie Wang, Alexander Schaffer, Shatabdi Roy-Chowdhury, Nadia A. Zatsepin, Andrew Aquila, Jesse Coe, Cornelius Gati, Mark S. Hunter, Jason E. Koglin, Christopher Kupitz, Garrett Nelson, Ganesh Subramanian, Thomas A. White, Yun Zhao, James Zook, Sébastien Boutet, Vadim Cherezov, John C. H. Spence, Raimund Fromme, Uwe Weierstall and Petra Fromme 
Table S1 Data quality statistics for phycocyanin.

\begin{tabular}{ccccccc}
\hline $\begin{array}{c}\text { Resolution } \\
(\AA)\end{array}$ & \#Reflections & \#Measurement & Multiplicity & $\begin{array}{c}\text { Completeness } \\
(\%)\end{array}$ & CC* & $\begin{array}{c}\text { SNR* } \\
\text { (CrystFEL) }\end{array}$ \\
11.04 & 1013 & 1154949 & 1140.1 & 100.00 & 0.989 & 7.90 \\
5.99 & 980 & 636800 & 649.8 & 100.00 & 0.981 & 5.67 \\
5.02 & 959 & 558792 & 582.7 & 100.00 & 0.983 & 6.00 \\
4.48 & 950 & 517920 & 545.2 & 100.00 & 0.983 & 6.37 \\
4.11 & 939 & 422140 & 449.6 & 100.00 & 0.978 & 5.87 \\
3.85 & 951 & 322650 & 339.3 & 100.00 & 0.976 & 5.01 \\
3.64 & 952 & 264625 & 278.0 & 100.00 & 0.969 & 4.36 \\
3.47 & 931 & 211411 & 227.1 & 100.00 & 0.959 & 3.81 \\
3.33 & 951 & 145805 & 153.3 & 100.00 & 0.956 & 2.88 \\
3.20 & 940 & 100473 & 106.9 & 100.00 & 0.907 & 2.45 \\
3.10 & 925 & 85798 & 92.8 & 100.00 & 0.876 & 2.11 \\
3.01 & 970 & 77473 & 79.9 & 100.00 & 0.894 & 1.86 \\
2.92 & 911 & 59827 & 65.7 & 100.00 & 0.866 & 1.55 \\
2.85 & 933 & 48409 & 51.9 & 100.00 & 0.802 & 1.43 \\
2.78 & 965 & 39337 & 40.8 & 100.00 & 0.802 & 1.26 \\
2.72 & 898 & 27798 & 31.0 & 100.00 & 0.737 & 1.12 \\
2.67 & 924 & 21450 & 23.2 & 100.00 & 0.603 & 0.99 \\
2.61 & 942 & 17822 & 18.9 & 100.00 & 0.574 & 0.82 \\
2.57 & 947 & 14732 & 15.6 & 100.00 & 0.640 & 0.80 \\
2.52 & 927 & 11574 & 12.5 & 100.00 & 0.487 & 0.83 \\
Overall & $\mathbf{1 8 9 9 0 8}$ & $\mathbf{4 7 3 , 9 7 8 5}$ & $\mathbf{2 5 0 . 6 7}$ & $\mathbf{1 0 0 . 0}$ & $\mathbf{0 . 9 7 1}$ & $\mathbf{3 . 1 9}$ \\
\hline
\end{tabular}




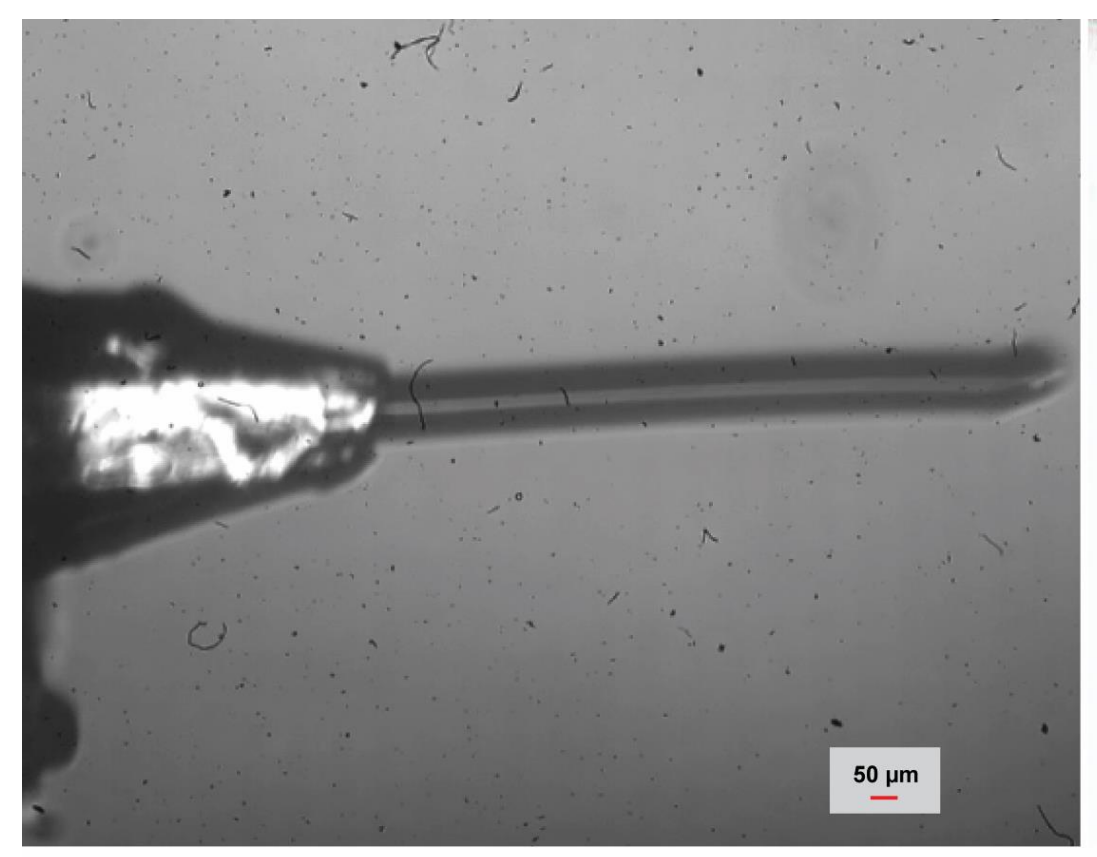

(a)

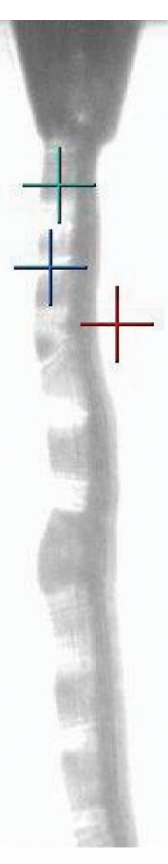

$75 \mu \mathrm{m}$

(b)

Figure S1 Agarose stream extrusion. (a) Agarose stream with no embedded crystals extruded in vacuum in a test chamber (b) agarose stream with embedded crystals extruded in vacuum in the CXI chamber. 


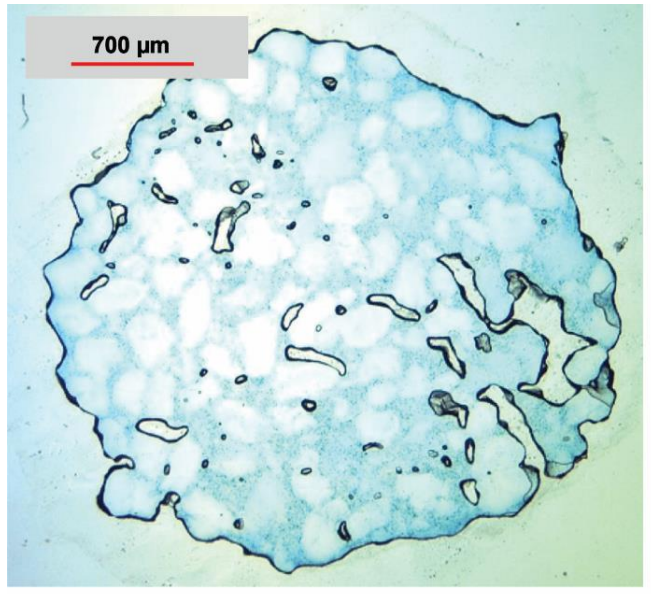

(a)

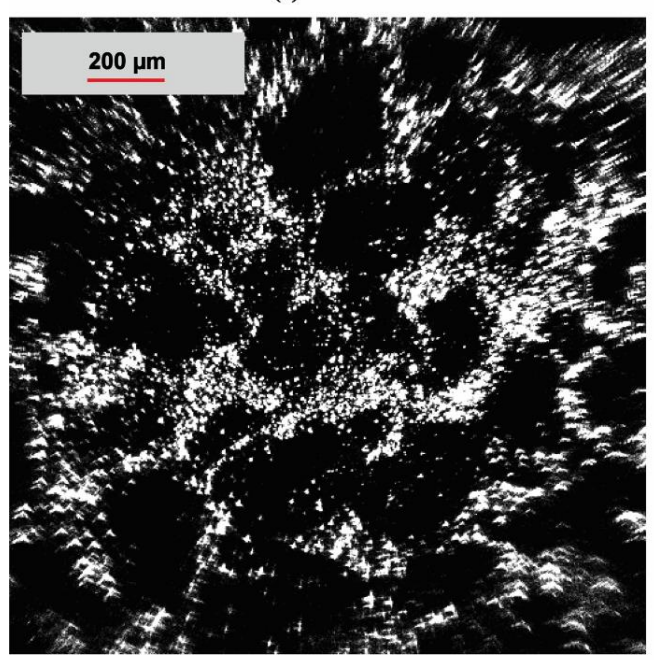

(b)

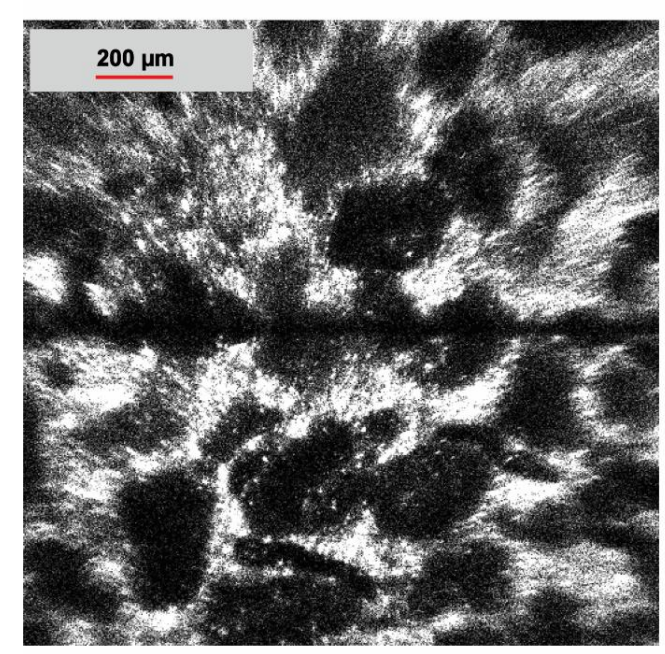

(c)

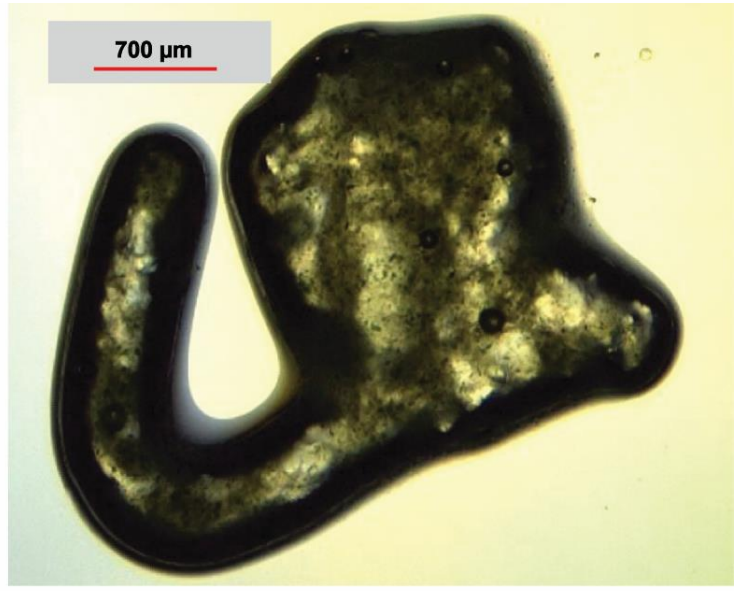

$(d)$

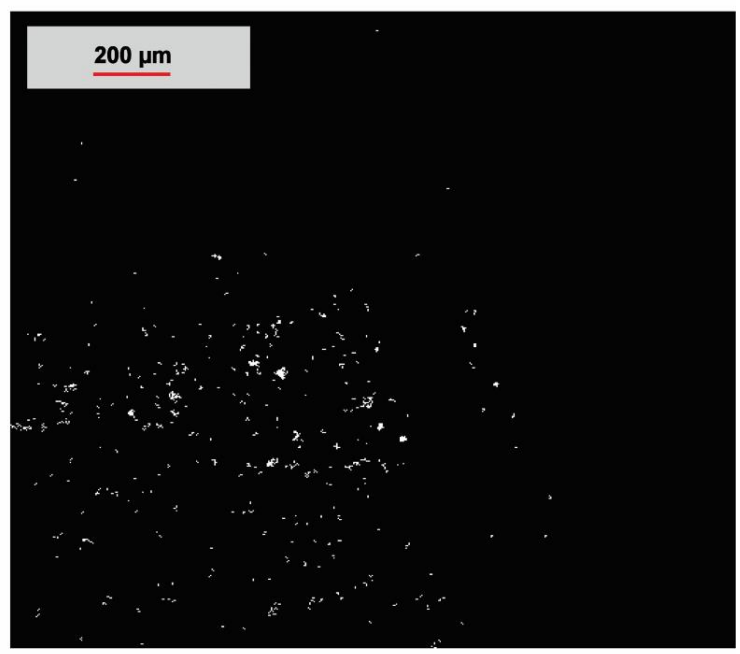

(e)

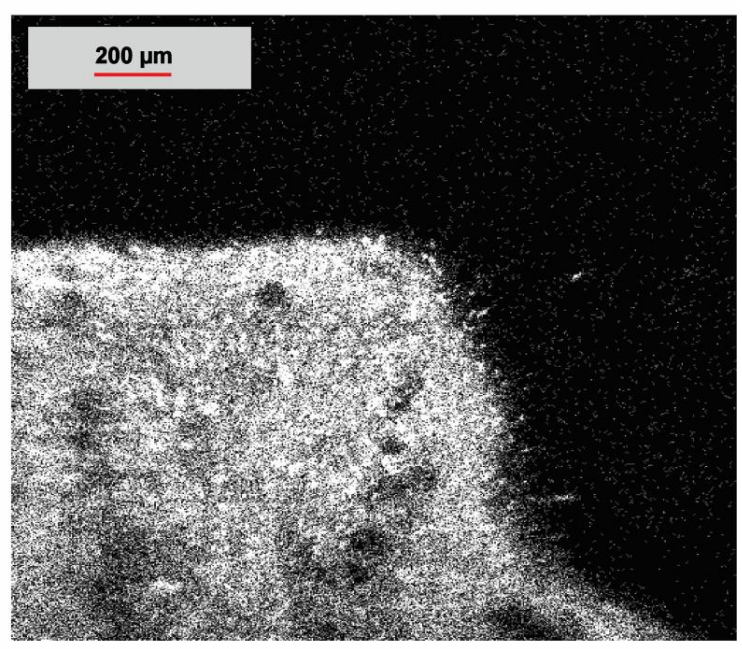

()

Figure S2 Characterization of PC and PSII embedded in agarose. (a) Brightfield of PC (b) second order non-linear imaging of chiral crystals of PC (c) ultraviolet two-photon excited fluorescence of PC microcrystals (d) brightfield of PSII (e) second order of non-linear imaging of chiral crystals of PSII (f) ultraviolet two-photon excited fluorescence of PSII. 

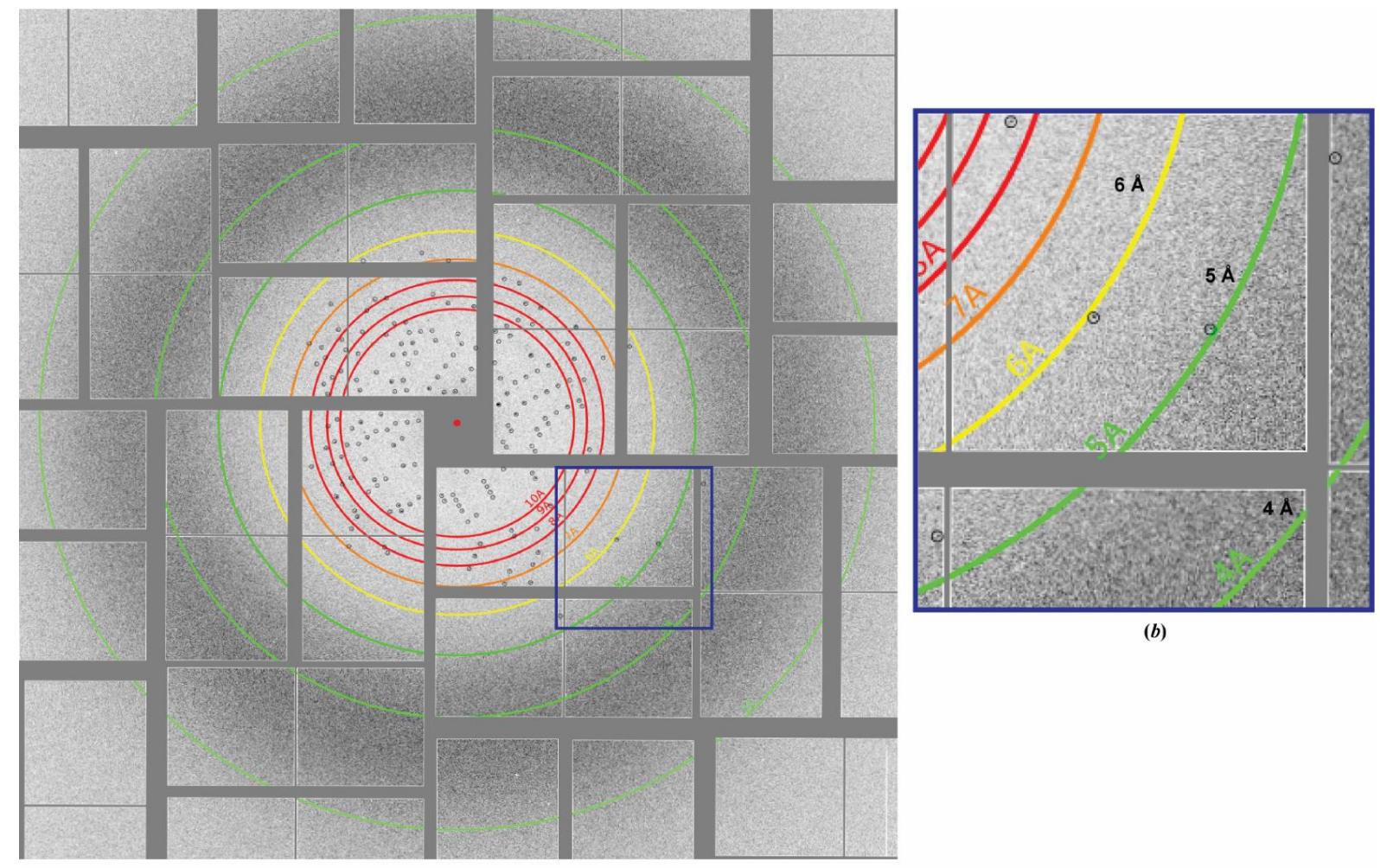

(b)

$(a)$

Figure S3 PSII embedded in agarose and delivered in a vacuum chamber. (a) Diffraction pattern from PSII in agarose, (b) magnified subset from (a).

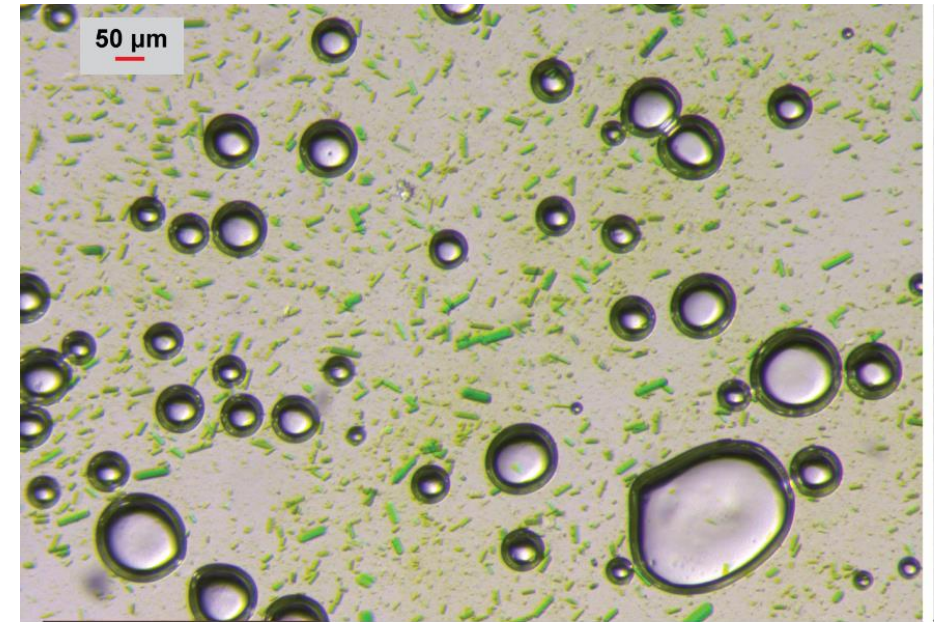

(a)

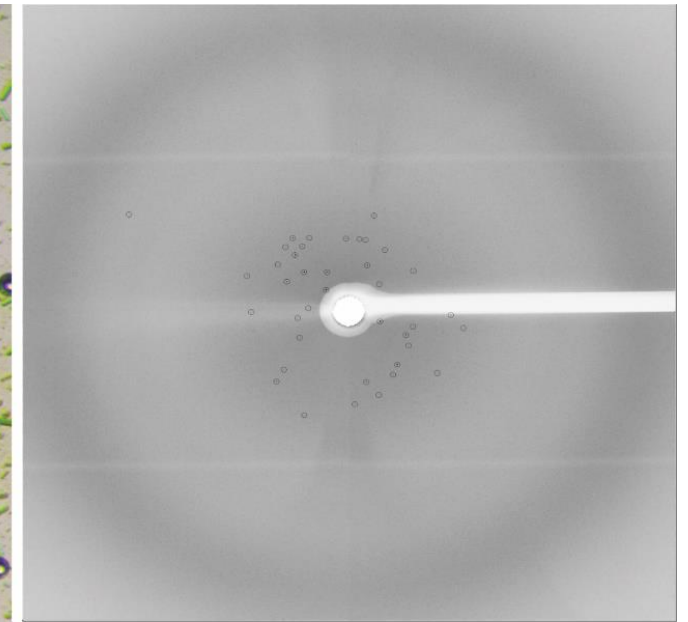

(b)

Figure S4 PSI embedded in agarose and delivered in a helium atmosphere. (a) Rod-like PSI crystals embedded in agarose, (b) diffraction pattern from PSI microcrystal in agarose. 